\title{
CONSIDERACIONES CRÍTICAS EN TORNO A LA CONSTITUCIÓN DEL MODO DE SER DEL ENTE. UN ESTUDIO A PROPÓSITO DE LA TESIS DE KANT: "EL SER NO ES UN PREDICADO REAL"
}

\author{
Mag. Daniel Armando Vivar Olivares ${ }^{1}$ \\ Pontificia Universidad Católica de Valparaíso, Chile
}

\begin{abstract}
Resumen: Este artículo discute el análisis que Heidegger hace respecto de la tesis kantiana "El ser no es un predicado real", el cual es desarrollado en Los problemas fundamentales de la fenomenología (1927). Allí se sostiene que el problema cardinal de la filosofía es el de la ontología. A la luz de una distinción esencial entre la filosofía como "concepción del mundo" y "filosofía científica", se hace patente, según Heidegger, el carácter científico de la filosofía, en tanto que su único problema es conducir a una clarificación del ser. De tal suerte, Heidegger pretende explicar la noción de existencia en vistas a una ontología fundamental. Él reconstruye el argumento kantiano para sugerir sus propias ideas y así describir, de una manera fenomenológica, el ser. Pero, creemos que esta descripción tropieza con algunos obstáculos propios de la distinción epistemológica clásica entre sujeto y objeto. De hecho, la formulación del problema de la existencia propulsado por Heidegger con vistas a una comprensión radical del ser, sigue prendada de la categoría de sujeto, puesto que es el Dasein (el individuo humano) quien, en última instancia, posibilita la comparecencia de todo ente.
\end{abstract}

Palabras claves: Ser $\cdot$ Kant $\cdot$ Heidegger $\cdot$ Dasein $\cdot$ Sujeto

Abstract: This article intends to discuss the analysis made by Heidegger when regarding the Kantian thesis "Being is not a real predicate", which he developed in The fundamental problems of phenomenology (1927). Heidegger maintains that the cardinal problem of philosophy is that of ontology. According to Heidegger, in the light of an essential distinction between philosophy as "the conception of the world" and "scientific philosophy", the scientific character of philosophy becomes clear, while its only problem is to lead to a clarification of being. In this way, Heidegger tries to explain the notion of existence in view of a fundamental ontology. He recreates the Kantian argument to suggest his own philosophical ideas, and thus to describe, in a phenomenological way, the being. But, we believe that this description encounters some obstacles which are typical of the classical epistemological distinction between subject and object. In fact, it seems that the formulation of the existence problem propelled by Heidegger with a view to a radical understanding of being still depends of the category of subject, since it is the Dasein (the human individual) who makes lastly possible the appearance of every being.

Keywords: Being $\cdot$ Kant $\cdot$ Heidegger $\cdot$ Dasein $\cdot$ Subject.

Enviado: 30/07/2018. Aceptado: 19/12/2018

\footnotetext{
${ }^{1}$ Magíster en Filosofía por la Pontificia Universidad Católica de Valparaíso. Candidato a doctor en filosofía. E-mail: danivivaroli@gmail.com
} 


\section{INTRODUCCIÓN}

En Los problemas fundamentales de la fenomenología, Heidegger examina cuatro tesis sobre el ser. En primer lugar, la tesis de Kant: el ser no es un predicado real. En segundo lugar, la tesis de la ontología medieval (escolástica) que se remonta a Aristóteles: a la constitución del ser de un ente le pertenece un qué [Was-sein] (essentia) y la subsistencia [Vorhandensein] (existentia). En tercer lugar, la tesis de la ontología moderna: los modos fundamentales del ser son el ser de la naturaleza (res extensa) y el ser del espíritu (res cogitans). Y, en cuarto lugar, la tesis de la lógica en el más amplio sentido: todo ente, con independencia de su modo de ser, puede ser encarado mediante el "es"; el ser de la cópula (Heidegger, 2000, pp. 40-41).

Este trabajo se ocupará de la primera tesis, a saber, el ser no es un predicado real, que, como se pudo ver, está asociada indudablemente a Kant. Su decantación está vinculada a la elaboración de la "diferencia ontológica", cuyo desarrollo será decisivo para el pensamiento heideggeriano, pero que explícitamente no aparece en Ser y tiempo.

Será propósito de este trabajo, entonces, presentar apropiadamente la tesis de Kant, de manera que la misma pueda ser desplegada desde sus distintas aristas, evitando, de este modo, soslayar elementos significativos para la discusión. En la misma línea, será preciso reconstruir la estrategia argumentativa de Heidegger para abordar dicha tesis, pues cabe señalar que pondrá en diálogo su propio entramado sistemático y filosófico con la tesis elaborada por Kant. Finalmente, será menester destacar ciertos puntos problemáticos en la lectura heideggeriana relativos a la constitución del modo de ser del ente, cuyo fundamento reside en el individuo humano.

En virtud de estos tres ejes se extraerán ciertas consideraciones críticas relativas a la condición aporética en la que se hunden los presupuestos tanto epistemológicos como ontológicos que conciernen a la constitución del Dasein. La tesis del presente escrito se apoya en la idea de que el sujeto elaborado en la modernidad, tanto por la filosofía cartesiana como por la kantiana, ha seguido filtrándose implícitamente allí donde se creía superado. En este sentido, la tesis proseguida por Heidegger respecto de Kant no avanza, creemos, teóricamente respecto del problema del ser y su constitución fundamental, pues la inmanencia inherente al sujeto moderno es homologable al Dasein en tanto éste es el último y fundamental eje en la relación intencional que supone el desocultamiento del ser del ente. 


\section{LA TESIS DE Kant: "El SER NO ES UN PREDICADO REAL"}

Kant expone su tesis a propósito de la prueba de la existencia de Dios en La Crítica de la razón pura (Dasein-Existenz,) (Kant, 2006, pp. 500-506). ${ }^{2}$ Ésta se remonta a San Anselmo, quien argumenta que a partir del concepto de Dios se concluye su existencia, pues, y aunque Anselmo no lo diga expresamente en estos términos, al ens perfectissimum no le puede faltar ninguna determinación positiva. Ahora bien, que una disquisición sobre la existencia de Dios esté inexorablemente ligada al problema del ser en general, a Heidegger no le parece extraño, pues este tipo de vínculo se remonta a la tradición de la ontología antigua y escolástica en la cual Kant está inserto:

"Estamos ante el hecho extraño de que Kant exponga el concepto más general del ser allí donde trata de la cognoscibilidad de un ente muy determinado, de Dios. Pero para quien conozca la historia de la filosofía (ontología), este hecho, lejos de serle extraño, le pone de relieve cómo Kant se inserta directamente en la gran tradición de la ontología antigua y escolástica. Dios es el ente supremo, summum ens, el ser perfectísimo, ens perfectissimum. Lo que es perfectísimo, es, evidentemente, lo más apropiado, en tanto que ente ejemplar, para que quepa extraer de él la idea de ser" (Heidegger, 2000, p. 55). ${ }^{3}$

En cualquier caso, más allá de que Kant no discuta que, de acuerdo con su concepto, Dios sea el ente más perfecto y que exista, —cuestión que para Heidegger pasa a segundo plano- su punto de ataque estriba en que la existencia no puede ser atribuible o perteneciente a la determinación de un concepto. Dicha determinación debe agregar algo al concepto que éste no tenía y de este modo aumentarlo. En este caso el ser o la existencia no serían determinaciones "reales" de la cosa. Por lo tanto, ser no es un predicado real, no es predicado de una res:

“Si tomo el sujeto (Dios) con todos sus predicados (entre los que se halla también la
omnipotencia) y digo 'Dios es', o 'Hay un Dios', no añado nada nuevo al concepto
de Dios, sino que pongo el sujeto en sí mismo con todos sus predicados, y lo hago
relacionando el objeto con mi concepto. Ambos pueden poseer exactamente el

${ }^{2}$ Heidegger precisa la distinción terminológica de Kant mostrando que en la refutación de este último al argumento anselmiano el concepto de Dasein/Existenz hace referencia a la subsistencia (Vorhandenheit) como efectividad. Por el contrario, en la filosofía heideggeriana Dasein será entendido como aquel ente que somos nosotros en cada caso, es decir, el individuo humano.

3 "wir stehen von der auffalenden Tatsache, da $\beta$ Kant den allgemeinsten Begriff des Seins überhaupt dort erörter, woer von der Erkennbarkeit eines ganz bestimmten, ausgezeichneten Seienden handelt, von Gott. Aber wer die Geschichte der Philosophie (Ontologie) kennt dem ist die Tatsache so wenig befremdend, da $\beta$ sie gerade deutlich macht, wie unmittelbar Kant in der großen Tradition der antiken und scholastischen Ontologie steht. (...) Diese Orientierung der Ontologie an der Gottesidee wurde für die nachkommende Geschichte der Ontologie und ihr Geschick von ausschlaggebender Bedeutung" (Heidegger, GA 24, pp. 37-38). 
mismo contenido. Nada puede añadirse, pues, al concepto, que sólo expresa la posibilidad, por el hecho de concebir su objeto (mediante la expresión él es) como absolutamente dado. De este modo, lo real no contiene más que lo posible. Cien táleros reales no poseen en absoluto mayor contenido que cien táleros posibles" (Kant, A599/B627).

Hasta aquí el argumento no se explica cabalmente, por lo que se hace necesario introducir una requisitoria respecto de la nomenclatura kantiana. Ésta descansa en la aclaración terminológica del concepto de realidad de la cual depende la explicación y comprensión de la tesis. He aquí su explicitación.

El concepto de realidad apunta a los contenidos quiditativos de la cosa, es decir, al qué de una cosa, res. Se trata de las esencias y de las cosas posibles, de aquello que las cosas son, independientemente de si son efectivas o no. El ser, en este sentido, no responde a lo que la cosa es. Se podría decir, no responde a las determinaciones entitativas:

“...el ser no puede ser tratado al modo en que sí son tratados otros predicados cualesquiera. El ser no es una perfección como pretendieron los racionalistas. Desde el punto de vista del concepto de la cosa, ella no se ve aumentada. Como expresivamente dice Bennet: "si le pido a usted que me traiga una cerveza, y luego añado ... 'fría', eso puede hacer más difícil cumplir lo pedido; pero si añado 'existente', la tarea de usted no se ha vuelto más difícil, porque una cerveza existente sólo es una cerveza” (Neumann, 1995, p. 125).

Siguiendo esta lógica, Heidegger divide la tesis en una parte negativa y otra positiva. La negativa, el ser no es un predicado real, ${ }^{4}$ no representa un problema para Heidegger, pues, a todas luces, ve allí el hecho de que el ser no es un ente. Sin embargo, la parte positiva, en la cual Kant señala que la existencia es posición absoluta (percepción), le va a parecer oscura y ambigua, por lo que una aclaración en grado mayor parecería lo más adecuado:

“Así, del análisis provisional de la interpretación kantiana de la existencia, se siguen dos cosas. Primero, esta interpretación no sólo es oscura y, por ello, necesitada de

\footnotetext{
${ }^{4} \mathrm{Al}$ respecto es menester nuevamente servirse del análisis que desarrolla Hardy Neumann en lo tocante a esta cuestión: "Si, en términos latos, el predicado representa algo que decimos de alguna cosa, tal parece que al indicarnos Kant que este predicado no es real, cuando lo utilizamos como tal en referencia a una cosa, el resultado es no haber dicho nada sobre ella, porque el predicado en cuestión no es real. Pero no es esto lo que el filósofo quiere decir, pues cuando pretendemos hacer uso del ser como de un predicado, el ser abandona eo ipso esa condición para pasar a funcionar de otra manera. (...) Que el ser no sea un predicado real quiere decir, como se sabe, que aquél no es el predicado de una res. Y no sólo no es esto, sino que no es de ninguna manera una res o algún aspecto de ella" (Neumann, 1995, p. 124). Se puede ver con claridad en este argumento, tal como la ve Heidegger, la diferencia ontológica, en donde el ser no puede ser un género o una especie, etc, sino que es aquello en lo que todo se mueve y es, pero que no comporta en ningún caso la condición de un ente o de una res. El ser es, en terminología kantiana, la condición de posibilidad de todo ente.
} 
una claridad mayor; sino, segundo, es discutible incluso también en su forma más favorable de entenderla, en la que ser es igual a ser-percibido. ¿Debemos quedarnos en esta afirmación crítica negativa? Una crítica meramente negativa sería una empresa carente de valor frente a Kant y a la vez una ocupación infructuosa con relación a la meta que nos hemos propuesto" (Heidegger, 2000, p. 77).

La transparencia y claridad del argumento aclaratorio debe estar precedido por una retoma de los presupuestos conceptuales básicos que se hallan en el centro de la tesis misma. Para ello Heidegger se sirve de la vertiente fenomenológica.

De acuerdo con ella, establecerá un objetivo medular y, a la vez, una delimitación peculiar. Respecto de lo primero, Heidegger intentará una explicación fenomenológica del concepto de ser o de existencia, cuestión que como se sabe, constituye el nervio central de su obra y que será transversal, prácticamente, en todos sus escritos. Estos conceptos no han sido dados de suyo, por lo que requieren una necesaria explicitación y a su vez una detallada genealogía.

Ahora bien, la delimitación peculiar a la que se ha aludido está suscrita a un cambio de registro en el método propiamente husserliano respecto del que Heidegger quiere aplicar. Si la fenomenología husserliana está adscrita al análisis descriptivo de las vivencias intencionales (cf. Husserl, 1985, pp. 471-589), Heidegger no hará otra cosa que remitir su análisis fenomenológico al ser mismo, como tal:

"Debemos comprender el ser para estar en disposición de que se nos dé un mundo que es, para existir en él y para que nuestro propio Dasein que es pueda ser. (...) Debemos comprender el ser, ser que no puede ser ya denominado ente, ser que no puede aparecer como un ente entre los demás entes, pero que, sin embargo, debe darse y de hecho se da en la comprensión del ser [Seinsverständnis]" (Heidegger, 2000, pp. 35-36).

\section{HeIDEGGER: EL ARGUMENTO ACLARATORIO}

La intención, por parte de Heidegger, de aclarar positivamente el concepto de ser y existencia en su dimensión fenomenológica no va de la mano con una contraposición radical respecto de la tesis planteada por Kant. Por el contrario, Heidegger quiere proseguir el punto de arranque del propio Kant y no contraponer simplemente su opinión. Para ello, es necesario considerar la tesis filosófica de Kant y explicarla. Pero, esta explicación debe tener como objetivo la fijación del punto de partida y el horizonte desde donde emerge la tesis, pues si Heidegger quiere proseguir el argumento kantiano, lo hará, mostrando a su vez, el desarrollo de su propia filosofía. Así, por ejemplo, queda de manifiesto en el texto cuando señala:

"En definitiva, muy probablemente Kant se mueva en la dirección correcta en su intento de aclarar la existencia. Sólo que él no ha visto el horizonte a partir del cual y dentro del cual tiene que llevar a cabo la aclaración con la suficiente nitidez y 
esto porque no ha fijado previamente el horizonte y no lo ha preparado de manera expresa para su explicación" (Heidegger, 2000, p. 77). ${ }^{5}$

Respecto de lo dicho anteriormente, Heidegger (2000), pp. 44-49, expone su propio concepto de fenomenología. Y lo hace teniendo siempre presente que su finalidad última es una aclaración del concepto de ser y de existencia. Por eso, va a caracterizar a la fenomenología como método de la ontología, entendido este último como aquel que se refiere al ser como tal y no a los distintos entes:

\begin{abstract}
"Afirmamos, pues, que el ser es el verdadero y único tema de la filosofía. Esto no es un descubrimiento nuestro, sino que es una forma de plantear el asunto que surgió en el comienzo de la filosofía, en la antigüedad, y obtuvo su desarrollo más grandioso en la Lógica hegeliana. Ahora afirmamos meramente que el ser es el verdadero y el único tema de la filosofía. Esto quiere decir, negativamente, que la filosofía no es ciencia del ente, sino del ser, o, como suena la expresión griega, ontología" (Heidegger, 2000, p. 36).
\end{abstract}

Ahora bien, no se puede perder de vista que la posibilidad de la tematización del ser depende siempre de un ente. Pero se trata de un ente particular, el individuo humano, que somos nosotros en cada caso y que es vía de entrada para acceder al ser.

Dicho esto, Heidegger emprende el análisis propiamente fenomenológico del concepto de percepción en Kant en su intento por aclarar la noción de existencia, pues ha quedado de manifiesto, y el propio Heidegger conviene en esto, que el ser no es un predicado real (momento negativo). "No cabe tocar la tesis de Kant, en su contenido negativo, de que el ser no es un predicado real. Con ella, Kant dice fundamentalmente que el ser no es un ente" (Heidegger, 2000, p. 84)

Sin embargo, en la tesis planteada por Kant se consigna que ser es igual a mera posición (respectus logicus), y existencia es igual a posición absoluta (momento positivo) (Heidegger, 2000, p. 59).

Esta delimitación constituye para Kant el punto máximo de su análisis, pues más allá no se puede llegar. Existenz y Dasein expresarían, por tanto, una relación del objeto con la capacidad de conocer, cuestión que queda establecida en los "postulados del pensar empírico como tal” en la Crítica de la razón pura (Kant, A218/B265). Pues bien, en la misma medida, la discusión kantiana de los conceptos de Dasein y realidad se vinculan con el concepto de percepción, cuya significación no se nos ofrece directamente discernible.

\footnotetext{
5 "Vielmehr wollen wir Kants eigenen Ansatz,die Interpretation von Sein und Existenz, in seiner eigenen Blickrichtung weiter verfolgen. Am ende bewegt sich Kant sehr wohl in der rechten Richtung bei seinem Versuch, Dasein und Existenz aufzuklären. Nur sieht er den Horizont aus dem her und innerhalb dessen er die Aufklärung durchführen will, nicht hinreichend deutlich, und das deshalb nicht, weil er sich dieses Horizontes nicht zuror entsprechend versichert und ihnfür seine Erläuterung ausdrücklich bereitgestellt hat" (Heidegger, GA 24, p. 67).
} 
La posición absoluta, entonces, remite a la percepción. Pero, según Heidegger, como la percepción en Kant es algo que se muestra confuso y ambiguo, resulta imperioso mostrar una interpretación diáfana. He aquí el momento en que Heidegger comienza a instalar su entramado sistemático y el desarrollo de su propia filosofía, pues el fenómeno de la percepción, a decir nuestro, le proporcionará la palanca estratégica para fijar el despliegue de su teoría en lo tocante a la diferencia ontológica.

Pero, demos un paso más en la explicitación heideggeriana. La percepción, pues, admite una forma errónea de entenderla (Kant) y una forma correcta (Heidegger).

La forma errónea de concebirla consiste en determinar una relación entre dos cosas subsistentes, siendo la misma relación algo subsistente. Dicho de otro modo, el error estriba en suponer que, en la relación, un objeto le adviene a un sujeto como algo añadido. Esto, según Heidegger, yerra el conocimiento del fenómeno. Y esto es lo que, a Kant, quizás, no le permita ir más allá en su análisis.

La forma correcta, según Heidegger (2000), pp. 85-86, señala que el sujeto psíquico tiene una referencia intencional al objeto. Esto equivale a decir que un modo de ser del sujeto psíquico es esta relación intencional, de suerte que esta característica se constituye como un a priori del sujeto. La percepción misma es un estar remitido a objetos, sean estos de naturaleza real o ilusoria.

Ahora bien, en esta relación el carácter de percibido (ser-percibido) apunta al hecho de que un objeto pueda, ciertamente, ser percibido. Este rasgo esencial, no le pertenece al objeto como tal, sino al Dasein. Si el modo de ser del ente, en un primer momento, determina el modo de descubrir el ente, será, en definitiva, el Dasein el que posibilite que las cosas se presenten en su modo de ser particular. ${ }^{6}$ El Dasein, en sentido estricto, deja que el ente comparezca por sí mismo. Lo saca de su ocultamiento. Pero, sólo habiendo el Dasein comprendido la condición abierta y ex-tática que se halla en su constitución fundamental, puede descubrir, a su vez, el modo de ser del ente y determinar su realidad o subsistencia.

En esta problematización de la tesis kantiana se avizora una aproximación fundamental que remite a la diferencia entre ser y ente, como ya habíamos mostrado. ¿Por qué? Porque la comprensión previa del ser, inherente al Dasein, permite una apertura radical en la que el ente se descubre. Dicho en tono heideggeriano: "El ser funda la posibilidad del estar-descubierto el ente"

\footnotetext{
${ }^{6}$ Consignemos que Heidegger sostiene que al único ente al que le va su ser es al Dasein. De lo cual se desprende que el descubrir el modo de ser de los distintos entes, está posibilitado por la "percepción" que libera y descubre al ente para que se presente el mismo. (Heidegger, 1997, pp. 25-37).
} 
(Heidegger, 2000, p. 103). Y es esta respuesta la que Kant, insinuándola, no pudo dar. ${ }^{?}$

Todo lo mostrado hasta aquí por Heidegger parece, a primera vista, un examen riguroso y meticuloso en su intento por alcanzar una ontología fundamental. Sin embargo, algunos aspectos claves dentro de su entramado parecen contradictorios y no escapan a la invectiva que el mismo parece utilizar.

\section{El PROBLEMA DEL SUJETO}

La escisión epistemológica fundamental entre sujeto y objeto ha sido el campo demarcatorio de la filosofía moderna, particularmente desde Descartes (1995, p. 27) hasta Kant (A1/B2). ${ }^{8}$ Debido a ello se ha intentado establecer criterios normativos y legales con el fin de sentar, científicamente, las bases y condiciones de posibilidad del conocimiento. La emergencia del concepto de sujeto ha posibilitado férreamente la construcción de la realidad en tanto en cuanto esta última depende de las fuerzas cognoscitivas que se hallan en el primero. De tal suerte, los objetos, notas o estímulos, le advienen a un sujeto que tendrá que filtrar, organizar y delimitar, según sus propias condiciones espaciotemporales, aquello que en un "afuera" se presenta como nebuloso. Respecto de lo anterior estima Kant que: “(...) el tiempo no es más que una condición subjetiva de nuestra (humana) intuición (que es siempre sensible, es decir, en la

\footnotetext{
${ }^{7}$ Desde el punto de vista kantiano es posible esgrimir, adelantándonos, un argumento en lo tocante a la estrategia argumentativa de Heidegger. Si Kant insinúa una respuesta al respecto, pero no da con el meollo de la cuestión, es porque previamente, y bajo una "limitación arbitraria", Heidegger ha fijado su objetivo último. Desde esta perspectiva el análisis heideggeriano estaría determinado por aquello que hace posible el despliegue de su filosofía, a saber, su estrategia argumentativa. Ésta reconduce en última instancia a la diferencia ontológica que es adonde Heidegger quiere llegar. Debido a esto último, la estrategia funcionaría como un constructo previo respecto del cual Heidegger irá insertando elementos que sean coherentes con su sistema. De ahí que bajo cierta unilateralidad de los argumentos kantianos Heidegger crea superar el punto donde Kant "inevitablemente" se detuvo. Pero, además, es necesario señalar que cuando Kant analiza el problema de la existencia (posición absoluta/percepción) lo hace con vistas a una relación en la que el objeto le adviene a un sujeto, siendo este último el que añade la fuerza cognoscitiva. En este sentido, y con vistas a la aclaración del ser, Heidegger, por así decirlo, "no renueva" el esquema kantiano, en tanto la relación intencional bajo la cual se descubre el modo de ser del ente, no es sino una reconducción hacia un "sujeto" que posibilita la aparición o el descubrir del ente.

${ }^{8}$ En el contexto filosófico denominado modernidad la pareja racionalismo-empirismo exponía las dos fuentes del conocimiento humano. Se trataba de una disputa epistemológica que ubicaba, por un lado, a la razón como fuente fundamental del conocimiento humano y por el otro a los sentidos o mundo empírico como primera aproximación al conocimiento humano. Kant sintetiza estas dos posiciones asumiendo que una primera aproximación se da, precisamente a través de los sentidos, pero que este mismo material empírico depende de las condiciones subjetivas que posee la razón. Sin ella el conocimiento no sería posible. Así, puede leerse en algunos pasajes de la Crítica: "No hay duda alguna de que todo nuestro conocimiento comienza con la experiencia" (B1). Cf. Kant, 2006, p. 41. Y más adelante: "Pero, aunque todo nuestro conocimiento empiece con la experiencia, no por eso procede todo él de la experiencia" (B2). Cf. Kant, 2006, P. 42).
} 
medida en que somos afectados por objetos) y en sí mismo, fuera del sujeto, no es nada" (A35/B51).

Esta pre-suposición constituye el punto álgido de una filosofía de lo anterior, de lo antepuesto y de los acuerdos tácitos que la razón ordenadora se da a sí misma, y desde la cual emerge el sujeto moderno como una sustancia idéntica e inmutable (Heidegger, 1998). Es más bien, una filosofía de lo previo que, sin encontrarse nunca con la certeza absoluta, al menos la condiciona.

Ahora bien, esta deriva conceptual continúa filtrándose allí donde se creía superada.

En este sentido, Heidegger cree poder desligarse, tanto de la onto-teología como de las filosofías apoyadas en la división antes mencionada. No sin razón ha operado una destrucción (Abbau) de la metafísica, es decir, un desmantelamiento de los presupuestos básicos que organizaban la tradición filosófica occidental. Por ello, resulta extraño cuando, Heidegger, mostrando cuál es el modo correcto de concebir la percepción lo haga, a pesar suyo, con vistas a un sujeto. Entonces resulta atingente preguntarse ¿A qué nos referimos con el concepto de sujeto? y ¿En qué medida puede existir cierta homologación con aquello que Heidegger denomina Dasein?

Cuando hablamos de sujeto lo hacemos con vistas a ese discurso que considera a esta noción desde una óptica, por así decirlo, sustancialista, según la cual se ha pretendido brindarle una cierta consistencia y primacía epistemológica. Así, el descubrimiento moderno del cogito cartesiano introdujo la completa certeza, claridad, distinción y evidencia de un "yo" irreductiblemente clausurado:

"¿A qué aludimos con el nombre de sujeto? ¿Qué designa con él toda nuestra tradición filosófica sino el propio objeto del "descubrimiento" de Descartes - tenido por inaugural de los tiempos modernos-, es decir, descubrimiento de la certeza de sí de la conciencia reflexiva en cuanto se distingue, como sujeto pensante soberano, de sus objetos de pensamiento?" (Juranville, 1992, p. 91).

En este sentido el estatuto de la subjetividad comprendía ineludiblemente a la razón $\mathrm{y}$ al pensamiento como ejes productores del mundo y su contenido. A partir de esta noción, el sujeto occidental emergió bajo la forma de una unidad compacta y autotransparente, de una presencia esencial y positiva:

“(...) Me di cuenta de que mientras quería pensar de esta suerte que todo era falso, era preciso necesariamente que yo que lo pensaba fuese alguna cosa; y notando que esta verdad: Pienso, luego existo, era tan firme y segura (...) podía recibirla sin escrúpulo como el primer principio de la filosofía que buscaba" (Descartes, 1995, p. 27).

La seguridad y claridad de este principio quedan aseguradas por la intuición espontánea que el pensamiento tiene acerca de sí mismo. No existe otro 
principio que se manifieste tan clara y directamente como el del reconocimiento de la ipseidad. ${ }^{9}$

Precisamente, estas consideraciones modernas son la que para Heidegger equivocan el camino cuando se trata de pensar la esencia o el ser del hombre:

"Para descubrir la esencia o ser del hombre lo que no se puede hacer es considerarlo aislado y aparte, por sí y en sí mismo. (...) el pensar metafísico moderno no sólo ha hecho eso, sino que, además, ha fundamentado y radicado en el hombre, concebido como cogitatio, subjetividad, ego, el ser de las demás cosas. La experiencia moderna de lo ente, de las cosas, consistió en ponerlas como ob-jetos para y desde un subjeto; las cosas, en este modo de pensamiento, son real y efectivamente presentes cuando han sido retrotraídas a la representación, que las asegura y les da suelo firme; en la certeza de la conciencia las cosas adquieren un ser seguro y firme" (Soler, 2007, pp. 71-72).

Ahora bien, es indiscutible, y a la vez insólito, que Heidegger en el mismo momento en que pretende una desvinculación con el sujeto quede prendado de él.

Dice, por ejemplo: "El ser-percibido de algo subsistente no es, en sí mismo, subsistente, sino que pertenece al Dasein, lo que no quiere decir que pertenezca al sujeto y a su esfera inmanente" (Heidegger, 2000, p. 100). Se esperaría, después de lo señalado anteriormente, una aclaración radical, al modo de Heidegger, respecto de esta distinción. Y quizás sí lo haga, cuando determina que lo subsistente es encontrado por sí mismo toda vez que el ser-percibido pertenece al comportamiento intencional perceptivo. Pero, hemos de advertir que el propio Heidegger, aun cuando se esmere en aclarar que este carácter de la intencionalidad no tiene nada que ver con algo subjetivo u objetivo, sigue dentro de una lógica que no despeja esta duda. De hecho, suspende esa tarea señalando: "no solucionaremos ahora este problema (...)" (Heidegger, 2000, p. 154). Y no lo soluciona, creemos, porque sin duda la relación intencional que presupone el descubrir un ente está ya precedida y determinada por el Dasein. Y este descubrir, a su vez, es un liberar lo subsistente para que pueda venir al encuentro. Por lo que es menester preguntar: ¿Quién descubre? ¿Quién ejecuta el acto liberador?

Para responder a estas preguntas atendamos a la siguiente cita: "La comprensión preontológica del ser es la que funda la posibilidad de que los entes comparezcan en cuanto tales ante nosotros y, por consiguiente, la comprensión del ser, que es constitutiva del Dasein, es lo que hace posible, esto es, lo que fundamenta, la pregunta filosófica por el ser de los entes" (Rivera, 2007, p. 154). Entonces, si

\footnotetext{
${ }^{9}$ Esta coincidencia del sujeto consigo mismo le viene dada, también, por el concepto de conciencia que le es inherente: "La idealidad y la sustancialidad se relacionan consigo mismas, dentro del elemento de la res cogitans, por un movimiento de pura auto-afección" (Derrida, 2005, p. 130). En este sentido, el "afuera" estaría determinado de parte a parte por aquellos procedimientos autoafectivos -sean de la idealidad, la sustancialidad o de la conciencia-, que beben de las fuentes del "adentro" cuyo nombre moderno no es sino el de cogito.
} 
es el Dasein el que ejecuta el acto comprensor, descubridor y liberador: ¿En qué se distingue del sujeto, es decir, de una subjetividad apoyada en la certitud de su constitución fundamental posibilitante?

Detengámonos por un momento en la cita anterior y extraigamos sus posibles tesis y consecuencias. En primer lugar, se establece que los entes "comparecen" ante nosotros respecto de una comprensión preontológica del ser. Estas primeras líneas enuncian esta comprensión preontológica de un modo impersonal e indeterminado, pues se muestra el acto de la comprensión por el cual los entes se presentan ante nosotros, mas no el "ejecutor" de esa "posibilidad". De tal suerte, esta primera enunciación prepara el camino que determinará quién es el que ejecuta el acto comprensor. Consiguientemente, en la segunda parte del párrafo, se señala que es el Dasein el que hace "posible" la pregunta por lo entes, pues a él le pertenece "constitutivamente" la comprensión del ser.

En este sentido, el quién (Dasein) como ejecutor posibilitante de la comparecencia de los entes nos remite a un sujeto cuyo modo de ser se mantiene idéntico a sí mismo y desde el cual se hacen posibles los distintos modos de ser de los entes o de los objetos, pues es el "único" que comprende la aperturidad del ser. ${ }^{10}$

Entonces, si hemos visto que el concepto de sujeto remite a una cierta inmutabilidad, hemos de ver también que en la relación intencional que presupone el acto descubridor y liberador lo que se mantiene inmutable e incorruptible es el modo de ser del Dasein.

Sea como fundamento anterior, sea como constitución ulterior del modo de ser del ente que se le impone, el Dasein es lo que permanece idéntico a sí mismo en tanto posibilidad y aperturidad al ser como tal. Es decir, es el fundamento último y la sustancia inmutable que abre el campo de los modos de ser del ente y del ser en general. Incluso, y yendo aún más lejos, no hay posibilidad del ser sin el Dasein. El Dasein humano "pastorea" al ser. Por ende, este último en su radical comprensión depende del Dasein, del mismo modo que el objeto como re-presentación depende del sujeto: "El ser del sujeto (...) se define en función de un objeto manipulable constituido a la medida de su representación y de su certeza, igualmente representada" (Binetti,

\footnotetext{
${ }^{10}$ Sin embargo, en el $\S 13$ de Ser y tiempo, Heidegger aclara que el Dasein no es homologable al sujeto respecto de la relación que uno y otro pudiera mantener con un afuera (objeto; mundo). Allí, pone de relieve que el conocimiento es ya una forma primaria de ser del Dasein que, a su vez, puede ir alcanzando nuevos estados y nuevas formas de estar-en-el-mundo. De tal suerte, el conocimiento es un modo de existir que se funda en el estar-en-el-mundo, en cuyo caso, dice Heidegger, no hay commercium ni entre el sujeto con respecto al mundo, ni entre el mundo con respecto al sujeto. Se trata de una constitución fundamental y primaria que soslaya la posibilidad de una escisión epistemológica entre sujeto y objeto. No obstante, lo que nosotros queremos remarcar es el modo en que el ente comparece respecto del Dasein. Porque más allá de la estructura constitutiva del Dasein con el mundo, creemos que sigue existiendo un muro insalvable en el modo de ser del Dasein con respecto a los entes. Estos últimos siempre adquieren el estatuto de entes a través de la mirada, por decirlo así, que le proyecta el Dasein. Es el Dasein el que ve las cosas siendo.
} 
2007, p. 127). Lo mismo podríamos argumentar respecto del Dasein. Los modos de ser del ente aún mostrándose desde sí mismos están definidos y determinados en función de la posibilidad de apertura que primariamente se da en el Dasein comprensor. De ahí que podamos homologar, en este sentido, Dasein y sujeto, ya que en los dos conceptos subyace una primacía constitutiva, cuya consistencia radica en que desde ellos se abre el campo de los entes u objetos.

Por eso Heidegger le otorga un lugar privilegiado al Dasein en la pregunta por el sentido del ser, pues será respecto de su analítica que el ser mismo en general podrá hacerse comprensible en su radicalidad. “(...) sólo se nos revelará el sentido del ser en general mediante la comprensión explícita del ser del Dasein y, particularmente, de su aperturidad" (Rivera, 2007, p. 127). Es evidente aquí la reconducción heideggeriana hacia un sustrato desde el cual emergería toda posibilidad comprensora y toda aperturidad ontológica. Sin ir más lejos, se dice que el Dasein, es un ente "señero", aislado y, a la vez, destacado entre los demás entes:

"Y lo que lo hace sobresalir por encima de todos los demás entes es su modo propio de ser. Este modo propio de ser es, en definitiva, la comprensión preontológica, esto es, no teórica sino vital, del ser. (...) o sea, la pregunta por el sentido del ser en general surge de una primacía óntica del Dasein sobre los demás entes. Y eso nos obliga a dirigirnos primeramente al Dasein si queremos plantear realmente la pregunta por el sentido del ser" (Rivera \& Stuven, 2008, p. 63).

¿No se ve, acaso, algo así como una presencia que vuelve tautológicamente sobre sí misma y que retiene las condiciones mismas del "producir”? ¿No es, en definitiva, una transposición subjetiva la que está operando subrepticiamente en el concepto de Dasein?

Con todo, insistimos, la relación intencional no se ofrece por sí misma. $\mathrm{Su}$ producción depende en última instancia del Dasein. La percepción permite que el ente comparezca por sí mismo, pero ese comparecer debe estar remitido al Dasein quien en definitiva hace posible esta comparecencia. De ahí que la línea demarcatoria que traza Heidegger entre Dasein y sujeto se mantenga en lo nebuloso. Quizás, no se trate, como observa Heidegger, de una esfera inmanente, pero no se puede negar que si el estar-revelado el ser funda la posibilidad del estar-descubierto el ente, esta revelación se da siempre en el Dasein humano. Lo extraño aquí es esa preeminencia (más allá de la "apertura" pre-comprendida) que posee el Dasein en esta peculiar estructura intencional.

En conclusión, querríamos poner de manifiesto que, en el intento heideggeriano por determinar y aclarar la existencia con vistas a una ontología fundamental, se producen ciertas fisuras argumentativas que aquí hemos destacado. Este escrito ha pretendido sumariamente mostrar que en Heidegger se sigue filtrando la escisión fundamental que ha operado en filosofía entre sujeto 
y objeto, pues aun cuando, a primera vista, Dasein se distinga del concepto de sujeto, su tematización conduce a ubicarlo en un lugar privilegiado respecto de los demás entes.

\section{BIBLIOGRAFÍA}

Binetti, M. (2007): “Kierkegaard-Derrida y la reconstrucción del sujeto". Daímon, 40, pp. 119-130.

Derrida, J. (2005): De la gramatología. Trad. Oscar del Barco y Conrado Ceretti. $8^{\circ}$ ed. Buenos Aires: Siglo XXI.

Descartes, R. (1995): Discurso del método. Barcelona: Ed. Planeta DeAgostini

Heidegger, M. (1975): Die Grundprobleme der phänomenologie. Gesamtausgabe, Band 24. Frankfurt am Main: V. Klostermann.

Heidegger, M. (1998): “La época de la imagen del mundo”. En: M. Heidegger, Caminos de bosque. Madrid: Alianza.

Heidegger, M. (2000): Los problemas fundamentales de la fenomenología. Trad. Juan José García Norro. Madrid: Trotta.

Heidegger, M. (2015): Ser y tiempo. Trad. Jorge Eduardo Rivera. $5^{\text {a }}$ ed. Santiago de Chile: Ed. Universitaria.

Husserl, E. (1985): Investigaciones Lógicas. Trad. Manuel García Morente y José Gaos. Madrid, Alianza.

Juranville, A. (1992): Lacan y la filosofía. Buenos Aires: Nueva Visión.

Kant, I. (2009): Crítica de la razón pura. Trad. Pedro Ribas. México: Taurus.

Neumann, H. (1995): “El ser como posición”. Philosophica, 18, pp. 119-130.

Rivera J. \& Stuven, M. (2010) Comentario a Ser y Tiempo de Martín Heidegger. Vol. 2, Primera sección. Santiago de Chile: Eds. Universidad Católica de Chile.

Rivera, J. \& Stuven, M. (2008) Comentario a Ser y Tiempo de Martín Heidegger. Vol. 1. Introducción. Santiago de Chile: Eds. Universidad Católica de Chile.

Rivera, J. (2007) En torno al ser. Santiago de Chile: Brickle.

Soler, F. (2007), "Prólogo", en: Martín Heidegger: Filosofía, ciencia y técnica, J. Acevedo (ed.), pp. 53-88. Santiago de Chile: Ed. Universitaria. 